\title{
Temperature gradients in the core overshooting region
}

\author{
M. Godart
}

Institut d'Astrophysique et de Géophysique, Université de Liège, Belgium

\begin{abstract}
The term overshooting is used to describe two situations: the chemical mixing induced by the convective elements crossing the boundary given by Schwarzschild's criterion (overshooting), or, in addition to that mixing, the change in the temperature gradient in the overshoot region when convection is efficient enough (penetration; Zahn 1991). We show that for models with the same mass of the mixed central region the oscillation frequencies are sensitive to the kind of overshoot treatment adopted. This effect is especially obvious for SPB stars and is high enough to be detected by CoRoT long run observations. That would imply the possibility of disentangling penetration from overshooting.
\end{abstract}

\section{HR diagram and internal structure}

For a given value of the overshooting parameter, different overshooting treatments yield different convective and mixed core mass fractions. For instance, an overshooting parameter of $\alpha_{\mathrm{ov}}=0.2$ with the penetration treatment results in a mixed core $3 \%$ smaller than the computed one with the classic overshooting treatment. As a consequence, in the case of the classic treatment the main sequence evolutionary tracks are longer and more luminous than in the penetration case. The larger the overshooting parameter is, the greater the effect will be. In order to have the same mixed mass fraction we have to decrease $\alpha_{\text {ov }}$ down to 0.175 in models computed with overshooting.

\section{Effect on the frequencies}

We study the impact of different overshooting treatments on the oscillation frequencies in order to possibly use asteroseismology as a tool to disentangle penetration from overshooting at the top of a convective core. In the extra-mixed region, the Brunt-Väisälä (BV) frequency is positive in models computed with overshooting while it is zero in models computed with penetration. This difference matters for modes that propagate in the deep radiative interior. These include low-order $\mathrm{g}$ - and $\mathrm{p}$-modes, which are excited in $\beta$ Cep stars, and high-order g-modes, which are excited in SPB stars (Figs. 1 and 2).

Fig. 1 shows the difference in normalized frequencies for a $10 \mathrm{M}_{\odot}$ star along the main sequence phase. For the $\mathrm{g}_{2}$ mode, it is of the order of $1.3 \%$, which means $0.4 \mu \mathrm{Hz}$, larger than the frequency resolution of CoRoT $(0.08 \mu \mathrm{Hz})$. Fig. 2 represents the period spacings of typical SPB frequencies for models computed with overshooting and penetration. The asymptotic values of the period spacing $\left(\sim\left(\int(N / r) d r\right)^{-1}\right)$ are different by about $150 \mathrm{~s}$. Furthermore the discrepancies between the period spacing computed with different overshooting treatments are of the order of $1550 \mathrm{~s}$ at $P \sim 1.2 \times 10^{5} \mathrm{~s}$, which could be detected by CoRoT.

In conclusion, even if the overshooting parameters are chosen to produce overshooting and penetration models with the same central mixed mass fraction, the temperature gradient in their central regions is different. As a result g-mode and low order p-mode frequencies may be affected. These effects of overshooting treatments on the frequencies are within the reach of current asteroseismic observations. 


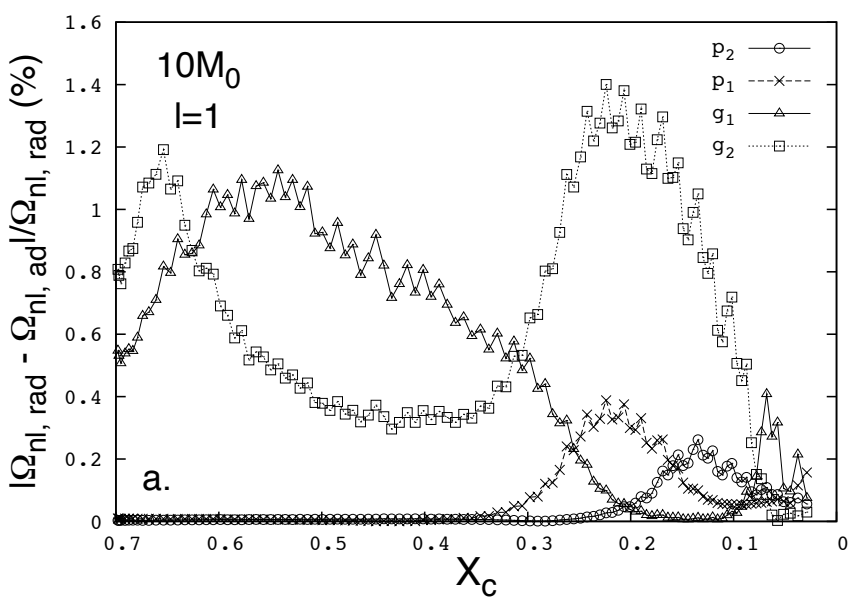

Figure 1: Difference between p-mode and g-mode normalized frequencies $\Omega_{n l}=2 \pi \nu_{n l} \sqrt{R^{3} / G M}(\ell=1)$ of modes for $10 \mathrm{M}_{\odot}$ stellar models computed with the different overshooting treatments.

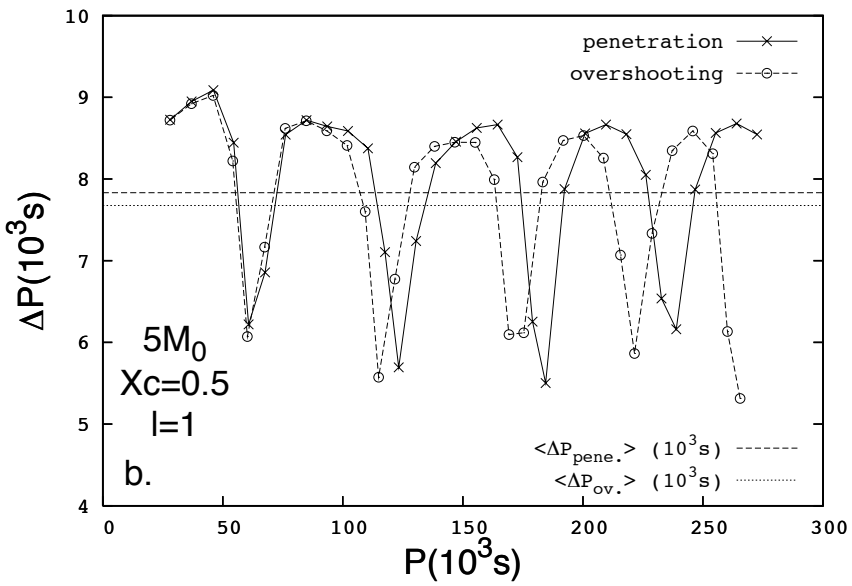

Figure 2: Period spacings $\left(\Delta P=\left|P_{n}-P_{n-1}\right|\right)$ for $5 \mathrm{M} \odot$ models computed with overshooting and penetration. $\langle\Delta P\rangle$ stands for the asymptotic value of the period spacing.

Acknowledgments. The author thanks J. Montalban for numerous fruitful discussions.

\section{References}

Zahn J.-P., 1991, A\&A, 252, 179 